\title{
Observation of Spin-Orbit Berry's Phase in Magnetoresistance of a Two-Dimensional Hole Anti-dot System
}

\author{
Ning Kang* and Eisuke Abe, Yoshiaki Hashimoto, Yasuhiro Iye, and Shingo Katsumoto \\ Institute for Solid State Physics, University of Tokyo, \\ 5-1-5 Kashiwanoha, Kashiwa, Chiba 277-8581, Japan
}

(Dated: February 6, 2020)

\begin{abstract}
We report observation of spin-orbit Berry's phase in the Aharonov-Bohm (AB) type oscillation of weak field magnetoresistance in an anti-dot lattice (ADL) of a two-dimensional hole system. An AB-type oscillation is superposed on the commensurability peak, and the main peak in the Fourier transform is clearly split up due to variation in Berry's phase originating from the spinorbit interaction. A simulation considering Berry's phase and the phase arising from the spin-orbit shift in the momentum space shows qualitative agreement with the experiment.
\end{abstract}

PACS numbers: 73.61.-r, 73.23.-b, 73.50.-h 
Spin-orbit interaction in two-dimensional system is predicted to introduce an additional geometric phase (Berry's phase) [1, 2, 3, 4, 5]. Here we consider the case of two-dimensional hole system (2DHS), in which the spin-orbit interaction is strong in III-V compound semiconductors due to the $p$-orbital nature of the valence bands. In a 2DHS with the Rashba-type spin-orbit coupling, the additional phase shifts arise in two ways. The spin-orbit interaction causes split of the parabolic dispersion, producing two Fermi circles of opposite spins (Fig[1(a),(b)) [6, 7, 8, 9, 10]. This means, the hole system has two different Fermi wave vectors, of which the difference $\left(\Delta k_{\mathrm{F}}\right)$ gives a phase shift and modifies the interference through, e.g., a ring structure. Another shift in phase comes from the spin part of the wavefunction. When a hole moves along a closed contour in the real space, the same occurs in $k$-space and it feels effective magnetic field $B_{\text {eff }}$ due to the spin-orbit interaction. $B_{\text {eff }}$ is in the $2 \mathrm{D}$ plane and orthogonal to the velocity. Hence when $B_{\text {eff }}$ is strong enough, the spin placed in the 2D plane turns by $2 \pi$ giving Berry's phase of 0 or $\pi$.

When an external magnetic field $B_{\text {ext }}$ is applied perpendicular to the 2DHS plane, the hole moving along a loop acquires an Aharonov-Bohm (AB) phase. At the same time, the spin gets some component perpendicular to the plane, which means some diminishing in the in-plane component (Fig.1(c)). This hence affects the above two additional phases. The variation in Berry's phase $\left(\Delta \theta_{\mathrm{B}}\right)$ and that in the phase due to $\Delta k_{\mathrm{F}}\left(\Delta \theta_{k}\right)$ are given as $11,2,3,4,4,12]$

$$
\begin{aligned}
& \Delta \theta_{\mathrm{B}}=\pi(1-\cos \theta), \\
& \Delta \theta_{k}=\pi r \Delta k_{\mathrm{F}} \sin \theta,
\end{aligned}
$$

where $\theta \equiv \arctan \left(B_{\text {eff }} / B_{\text {ext }}\right)$, and $r$ is the radius of the AB ring. Though significant warping of the outer Fermi contour is reported[6, 7] as shown in Fig.1(b), we can consider $\Delta k_{\mathrm{F}}$ as an average over the perimeter in the present discussion. Hence the phase of an $A B$ oscillation in a ring made of such a 2DHG should show continuous shift giving a nontrivial peak shape in its Fourier transform (FT) amplitude versus the frequency when the FT is performed in a finite field range. In experiments, such phases have been observed as splitting of $\mathrm{AB}$ magnetoresistance oscillation frequency in single $\mathrm{AB}$ rings [11, 12, 13]. Especially the authors of Ref. 12 observed nontrivial variation of the main FT peak due to the phase shift represented in Eqs.1] and 2.

In an anti-dot structure, the carriers are excluded from a dot region and magnetoresis- 
tance oscillation similar to that in an $\mathrm{AB}$ ring is expected. In an anti-dot lattice (ADL), $\mathrm{AB}$ oscillations of paths around single anti-dots should be averaged out because of random phasing, whereas the effect of the $\mathrm{AB}$ phase remains in the density of states resulting in magnetoresistance oscillation called AB-type oscillation[14, 15, 16, 17]. It is, then, of strong interest whether the effect of spin-orbit Berry's phase appears in the density of states responsible for the AB-type oscillation.

In this letter, we report observation of the predicted Berry's phase through clear splitting of the main peak in FT of the AB-type oscillation in the magnetoresistance of an ADL. The shift in $k$-space is independently estimated from the Shubnikov-de Haas ( $\mathrm{SdH})$ oscillation. Because the ADL automatically performs the disorder average, random specific effects in $h / e$ oscillation are suppressed and intrinsic information can be obtained.

An $(\mathrm{Al}, \mathrm{Ga}) \mathrm{As} / \mathrm{GaAs}$ heterostructure was grown by molecular beam epitaxy on a (001) GaAs substrate. Delta-doped Be layers produced a 2DHS with the hole concentration of $2.3 \times 10^{11} \mathrm{~cm}^{-2}$ and the mobility of $6.8 \times 10^{4} \mathrm{~cm}^{2} / \mathrm{Vs}$ at $4.2 \mathrm{~K}$. SdH oscillation in the magnetoresistance of the 2DHS without ADL was measured at $60 \mathrm{mK}$. From the plot of the peak index versus the inverse magnetic field, the concentrations of holes with smaller Fermi contour and larger one as $0.78 \times 10^{11} \mathrm{~cm}^{-2}$ and $1.56 \times 10^{11} \mathrm{~cm}^{-2}$ respectively. In this letter, we call the former and the latter as l-holes and h-holes, respectively. In the simple effective mass model, these two spin-split hole bands correspond to the two Fermi contours in Fig.1(a), though significant warping in h-hole band is reported in more realistic band calculation [6, 7]. The grown film was wet-etched into Hall bars with [110] current direction. Circular anti-dot shape is adopted and ADLs were defined by electron beam lithography and wet-etching to a depth of $50 \mathrm{~nm}$. An ADL is characterized mainly by the dot diameter $d$, the lattice structure, and the lattice period $a$. In this study, we designed a square lattice aligned along [110] ([110]) with $d=250 \mathrm{~nm}$ and $a=500 \mathrm{~nm}$ (see Fig.1(d)).

The sample was directly immersed in ${ }^{3} \mathrm{He}-{ }^{4} \mathrm{He}$ mixture in a mixing chamber of a dilution refrigerator and cooled down to $60 \mathrm{mK}$. $B_{\text {ext }}$ up to $6 \mathrm{~T}$ was applied by a superconducting solenoid. The resistance was measured in four-terminal configuration by standard lock-in technique with a frequency of $80 \mathrm{~Hz}$.

The solid line in Fig 2 shows the resistance of the sample as a function of $B_{\text {ext }}$ up to $0.5 \mathrm{~T}$. In higher field region, formation of the edge states and alignment of hole spins perpendicular to the plane bring about different physics. Therefore, we deal mainly with this low-field 
region. The magnetoresistance shows a clear commensurability peak marked as A by an arrow in the Fig,2, Generally such peaks in resistance appear when the carrier cyclotron orbits are commensurate with an ADL and localized in the lattice. For a circular Fermi contour, the classical cyclotron radius $R_{c}$ is expressed as

$$
R_{c}=\frac{\hbar k_{\mathrm{F}}}{e B_{\mathrm{ext}}}
$$

where the Fermi wavevector $k_{\mathrm{F}}$ is written as $\sqrt{4 \pi p}$ with the hole concentration $p$ (here we assume the spin degeneracy is lifted). $B_{\text {ext }}$ at peak position A in Fig, 2 can be well ascribed to localized orbits of l-hole encircling single anti-dot. For h-hole, as noted above, we should take into account the effect of warped Fermi contour in Fig.1(b). This effect will be discussed in a separate paper. For a small hump structure around $0.05 \mathrm{~T}$, we do not have any appropriate interpretation at present.

As shown in Fig,2, an oscillation with a short period superposed on the commensurability peak is visible. In order to extract the oscillating component, we subtract the background commensurability structure by fitting slowly-varying functions. An example of extracted resistance variation is shown with the broken line in Fig,2, which exhibits a clear fast oscillation. Here for the background subtraction, a 12th order polynomial for each set of 100 consecutive data points is adopted. Although the selection of fitting function affects the outlook of the oscillatory component, little is affected in the main and sub peak structures in the Fourier space.

The observed oscillations are nearly periodic in $B_{\text {ext }}$. The SdH oscillation is not visible in this field range due to the restriction of the space for free cyclotron motion. The FT amplitude of the extracted oscillation is displayed in Fig. The magnetic field range is taken from $-0.5 \mathrm{~T}$ to $0.5 \mathrm{~T}$. At the main peak around $50 \mathrm{~T}^{-1}$, clear splitting into three subpeaks, marked as A, A' and B', is observed. Sub-peak A has a shoulder structure at lower frequency marked as B. From these four splitting frequency positions, the position of the main peak structure is determined to be $52 \mathrm{~T}^{-1}$, where a sharp dip marked as $\mathrm{C}$ is located. If we measure the oscillation frequency with the magnetic flux on the circle with radius $a / 2$, position $\mathrm{C}$ just corresponds to the flux quantum $h / e$, confirming that the peak structure originates from the $\mathrm{AB}$ effect around single anti-dots through the modulation in the density of states.

As noted above, the selection of functional form for background subtraction does not affect 
the characteristics shown in Fig 3 as long as the fitting function is slowly varying versus $B_{\text {ext }}$. We have even performed direct FT without subtraction. Though the background variation results in enhancement of unphysical low frequency component, the splitting of the main peak structure still exists in the result, confirming the above result.

Another characteristic structure in Fig $\left[3\right.$ is a dip-peak structure around $110 \mathrm{~T}^{-1}$, which corresponds to about twice the frequency of the main peak, and originates from the orbits with the winding number $n=2$. The signal-to-noise ratio is not high enough to resolve detailed peak structure, nevertheless we may view it as a dip and two side peaks as indicated by arrows. Though there is no remarkable structure at the positions at around $n=3$, a surprisingly clear peak is observed at the position for $n=4$ as shown in the inset of Fig 3 .

Figure 4 displays the temperature dependence of the FT spectrum. The three peaks (A, A' and B') show almost identical temperature dependence as seen in the inset of Fig, 4, which can be fitted by the Dingle function,

$$
f(T) \propto \frac{\kappa T}{\sinh \kappa T}, \quad \kappa \equiv \frac{2 \pi^{2} k_{\mathrm{B}}}{\Delta E},
$$

where $T$ is the temperature and $\Delta E$ the energy difference between the peaks in the density of states modulation caused by the $\mathrm{AB}$ effect. The fitting gives the value of $\Delta E$ as $86 \mu \mathrm{eV}$. This is in similar order with other measurement in 2D electron systems[17]. The agreement in the temperature dependence manifests the three peaks have the same physical origin. There, we stressed the nature of the ensemble average in AB-type oscillations in ADLs, as opposed to the ordinary AB effect in single ring structure. The AB-type oscillation manifests the oscillatory fine structure of the density of state, and the temperature dependence of the oscillation amplitude can be explained by thermal broadening of the energy levels of the orbits.

We attribute the origin of the splitting to shifts in additional phases expressed in Eqs.1 and 2. In the simplest approximation the oscillatory resistance $\Delta R$ is written as the sum of four terms:

$$
\Delta R=\cos \left(n\left(\theta_{\mathrm{AB}}+\Delta \theta_{\mathrm{B}}\right)\right)+\cos \left(n\left(\theta_{\mathrm{AB}}-\Delta \theta_{\mathrm{B}}\right)\right)+\cos \left(n\left(\theta_{\mathrm{AB}}+\Delta \theta_{k}\right)\right)+\cos \left(n\left(\theta_{\mathrm{AB}}-\Delta \theta_{k}\right)\right)
$$

where $\theta_{\mathrm{AB}}$ is the $\mathrm{AB}$ phase given by $2 \pi B_{\text {ext }} \cdot \pi r^{2} /(h / e)$. Because the phases in Eqs.1 and 2 are not linear in $B_{\text {ext }}$, each term in Eq.5 is not sinusoidal oscillation and hence the FT spectrum strongly depends on the region of transformation. By the same token, the FT 
lineshape for higher harmonics $(n>1)$ is not simply $n$-times enlargement in frequency axis of the main peak structure.

In order for a qualitative comparison with experiment, we calculate the FT spectra of Eq.5 in the magnetic field ranging from -0.5 to $0.5 \mathrm{~T}$ with $\Delta k_{\mathrm{F}}=4.1 \times 10^{7} \mathrm{~m}^{-1}$, which is the value measured from the $\mathrm{SdH}$ oscillation, and $r$ is taken to be $a / 2$. We adopt $0.55 \mathrm{~T}$ for $B_{\text {eff }}$, which is reported for 2 DHSs with conditions close to the present ones [9]. The results are shown in Fig.5. Despite the crude approximation, the solid lines in Fig.5 bears striking resemblance to the experiment. For $n=1$, clear splitting into 4 sub-peaks is observed. With increasing $n$, the distribution of the peaks increases but the variation of the center peak structure is not monotonic. For $n=2$, there is a sharp dip at the center while only a broad peak exists for $n=3$. When $n$ is increased to 4 , the peak distribution still broadens though the center peak resharpens. These changes in $n=3$ and 4 explain the disappearance and reappearance of peak structure in the experiment.

As shown by the dotted lines in Fig, 5 , when there is no contribution of Berry's phase, the characteristics of the center peak are completely different. No splitting appears even for $n=2$; Rounding and sharpening for $n=3$ and 4 do not appear either. These results hence support the interpretation that the splitting of FT peaks is due to the phases in Eqs.1 and 2 arising from the spin-orbit coupling, added to the AB phase. Equation 5 is too crude an approximation for further quantitative discussion. Especially, in ADLs, orbits encircling anti-dots may vary their sizes with $B_{\text {ext }}$, which is smaller effect than Berry's phase and results in simple shift of peak positions. Shift of peak positions, e.g., for $n=4$ may be explained with this effect.

It should be remarked that the mean free path of the present $2 \mathrm{DHG}$ is about $600 \mathrm{~nm}$, which is much shorter than even the perimeter of a closed trace encircling a single anti-dot. This fact clearly emphasizes that the mean free path is essentially different from the phase coherence length, and also from the circumference of cyclotron orbit. The former difference has been repeatedly mentioned in literatures[18] while the latter manifests that the major origin of the scattering is impurity potential, which allows free space for ballistic cyclotron motions.

Another experimental fact that supports our interpretation is that the peak splitting in the FT spectra disappears in the AB-type oscillation in high fields, where the spin is fixed perpendicular to the plane and neither variation in Berry's phase nor in $\Delta k_{\mathrm{F}}$ occurs. This 
phenomenon will be reported in a separate paper.

In conclusion, we have observed spin-orbit Berry's phase through splitting of Fourier transform peaks in the Aharonov-Bohm type oscillation of weak field magnetoresistance in an anti-dot lattice of a two-dimensional hole system. A simple simulation with adopting parameters obtained from independent measurements shows fair qualitative agreement with the experimental observations.

The authors would like to thank K. Suzuki for help during this experiment. This work is supported by a Grant-in-Aid for Scientific Research from the Ministry of Education, Culture, Sports, Science, and Technology of Japan and also supported by Special Coordination Funds for Promoting Science and Technology.

* Electronic address: ning.kang@issp.u-tokyo.ac.jp

[1] D. Loss, P. Goldbart and A. V. Balatsky, Phys. Rev. Lett. 65, 1655 (1990).

[2] A. G. Aronov and Y. B. Lyanda-Geller, Phys. Rev. Lett. 70, 343 (1993).

[3] A. Stern, Phys. Rev. Lett. 68, 1022 (1992).

[4] T. Z. Qian and Z. B. Su, Phys. Rev. Lett. 72, 2311 (1994).

[5] Y. Meir, Y. Gefen and O. Entin-Wohlman, Phys. Rev. Lett. 63, 798 (1989).

[6] T. Ando, J. Phys. Soc. Jpn. 54, 1528 (1985).

[7] U. Ekenberg, and M. Altarelli, Phys. Rev. B 30, 3569 (1984).

[8] J. P. Eisenstein, H. L. Stormer, V. Narayanamurti, A. C. Gossard, and W. Wiegmann, Phys. Rev. Lett. 53, 2579 (1984).

[9] J. P. Lu, J. B. Yau, S. P. Shukla, and M. Shayegan, Phys. Rev. Lett. 81, 1282 (1998).

[10] Y. Iye, E. E. Mendez, W. I. Wang, and L. Esaki, Phys. Rev. B 33, 5854 (1986).

[11] A. F. Mopurgo, J. P. Heida, T. M. Klapwijk, B. J. van Wees and G. Borghs, Phys. Rev. Lett. 80, 1050 (1998).

[12] J.-B. Yau, E. P. De Poortere and M. Shayegan, Phys. Rev. Lett. 88, 146801 (2002).

[13] M. J. Yang, C. H. Yang, and Y. B. Lyanda-Geller, Europhys. Lett. 66, 826 (1998).

[14] D. Weiss, K. Richter, A. Menschig, R. Bergmann, H. Schweizer, K. von Klitzing, and G. Weimann, Phys. Rev. Lett. 70, 4118 (1993).

[15] See for review e.g. T. Ando, S. Uryu , S. Ishizaka and T. Nakanishi, Chaos, Solitons \& 
Fractals, 8, 1057 (1997).

[16] F. Nihey and K. Nakamura, Physica B 184 (1993) 398.

[17] Y. Iye, M. Ueki, A. Endo, and S. Katsumoto, J. Phys. Soc. Jpn. 73, 3370 (2004).

[18] e.g. see Y. Imry, Introduction to Mesoscopic Physics 2nd ed. (Oxford, 2001). 

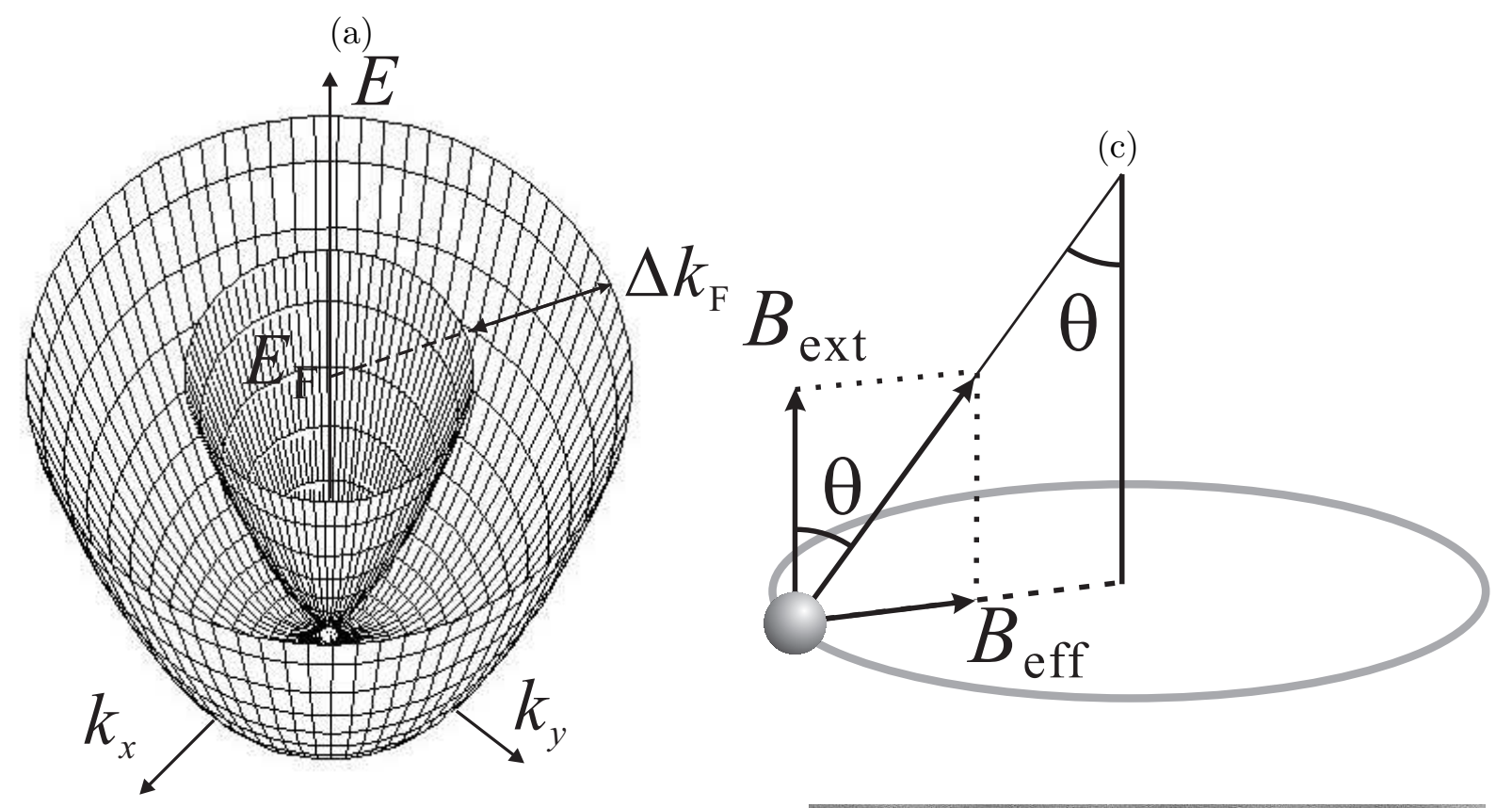

(b)
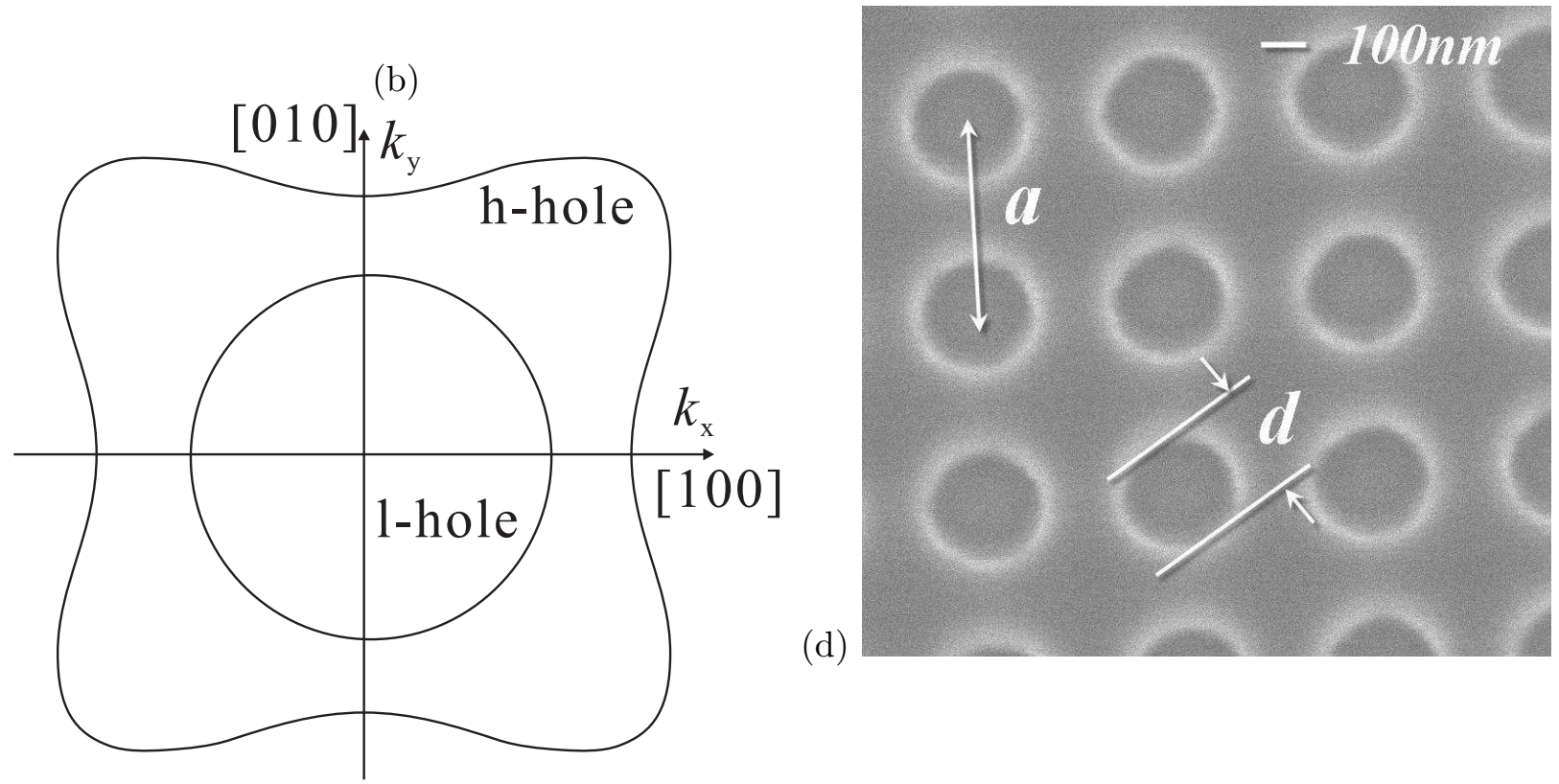

FIG. 1: (a) Surface plot of spin subbands explains two Fermi contours with different $k_{\mathrm{F}}$ appear as a result of spin-orbit interaction. (b) According to more realistic calculation [6, 7], the outer subband (h-hole) is significantly warped. (c) Schematic illustration of $B_{\text {eff }}$ seen by holes moving around a ring structure in the presence of spin-orbit interaction. (d) Scanning electron micrograph of the ADL sample. 


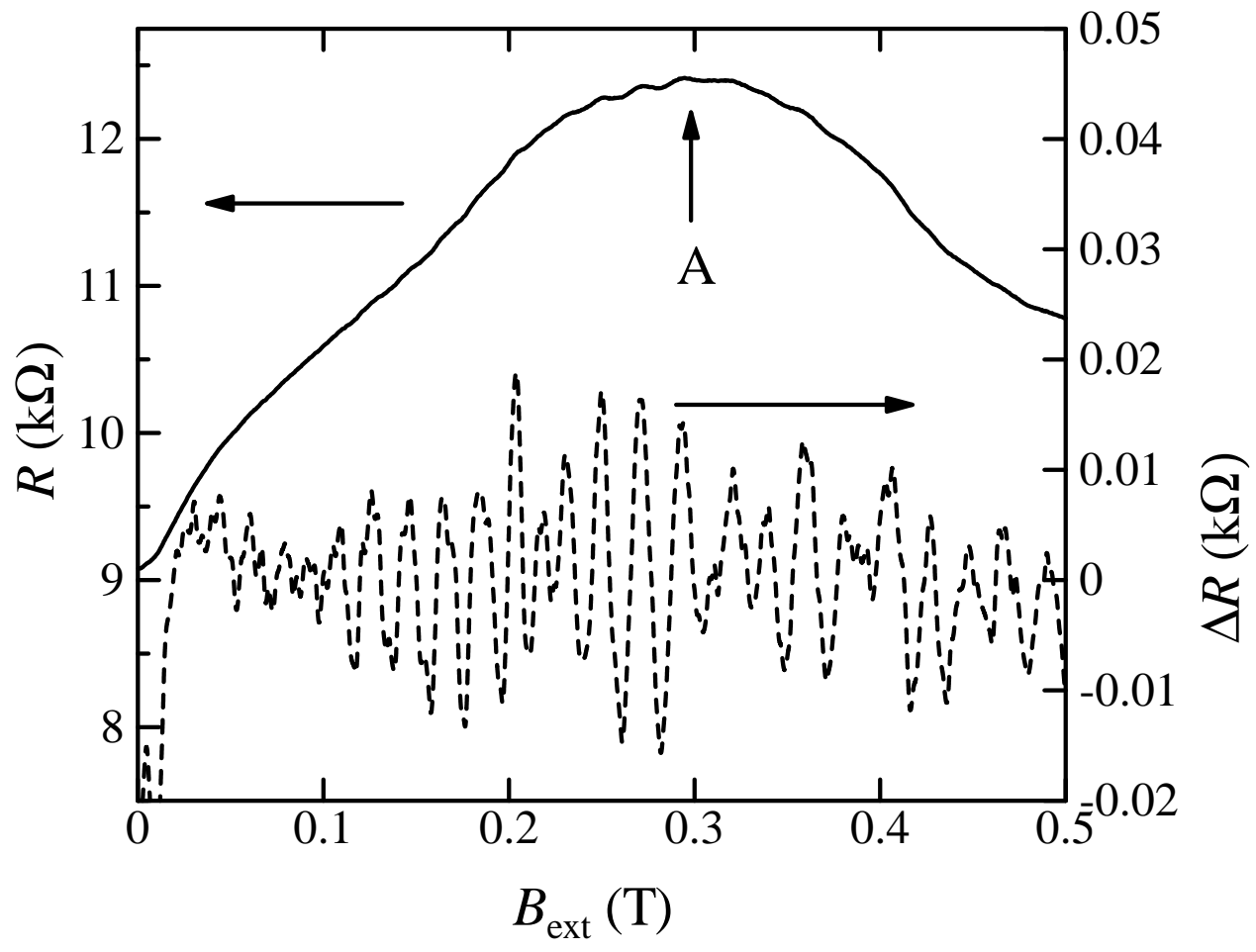

FIG. 2: Upper solid line: Resistance of the sample as a function of the external magnetic field $B_{\text {ext }}$ for $T=60 \mathrm{mK}$. Lower broken line: Extracted oscillating part of the magnetoresistance. For the background subtraction, a 12th order polynomial for each set of 100 consecutive data points is adopted. 


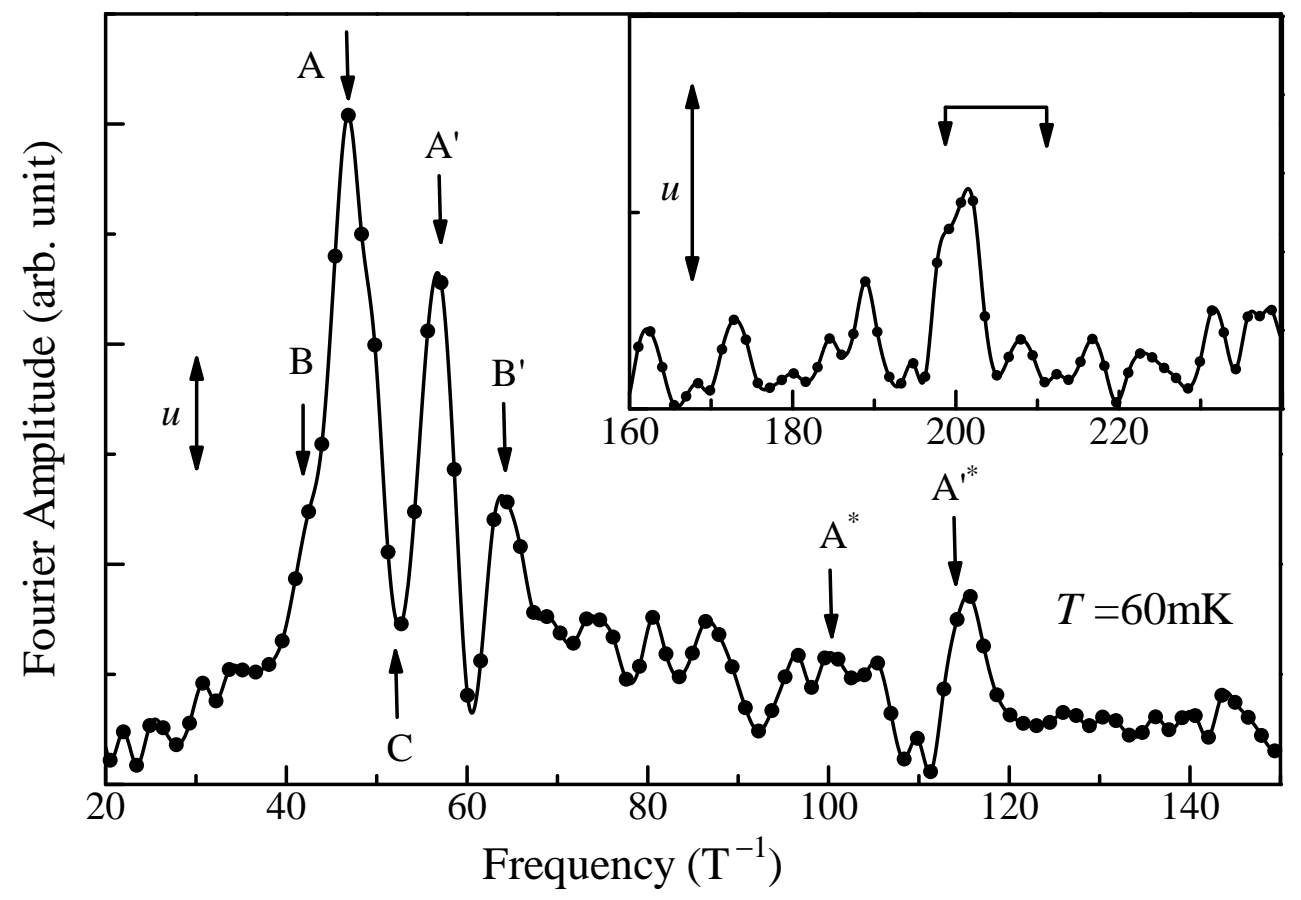

FIG. 3: Fourier spectrum of the AB-type oscillation in the magnetoresistance at $60 \mathrm{mK}$. The main peak splits into four (B, A, A', B') and also the 2nd harmonic splits into two $\left(\mathrm{A}^{*}, \mathrm{~A}^{* *}\right)$. (The other two are within the noise level.) The inset shows peak structures around four times frequency of the main peak structure as indicated by arrows. The scales of FT amplitude are shown by the arrows marked as $u$ in the respective figures. 


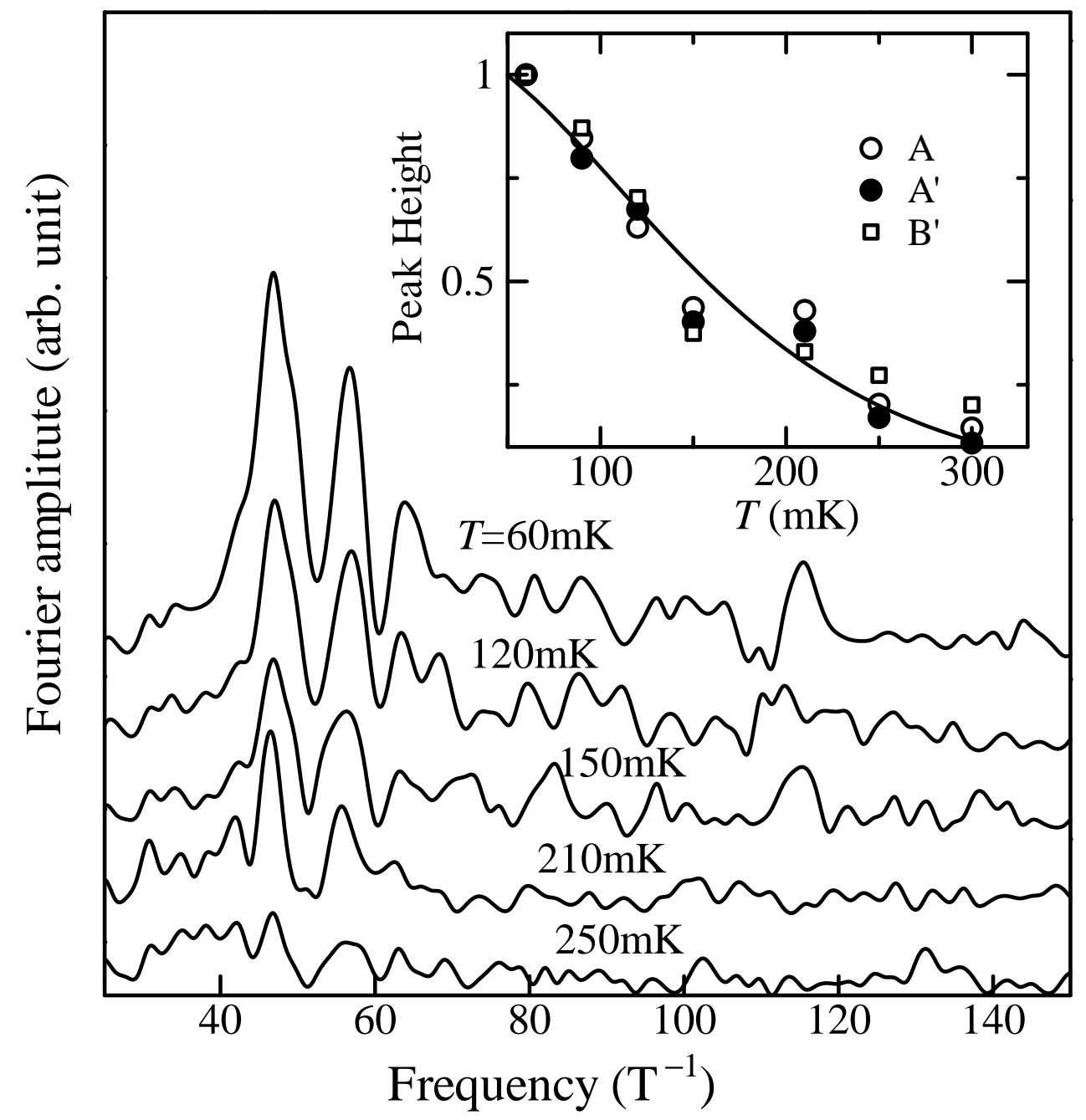

FIG. 4: FT results at temperatures from $300 \mathrm{mK}$ down to $60 \mathrm{mK}$. The data are offset for clarity. The inset shows the peak heights normalized at $60 \mathrm{mK}$ for peaks $\mathrm{A}, \mathrm{A}^{\prime}$ and B'. The solid line in the inset is the fit to the Dingle function (4). 


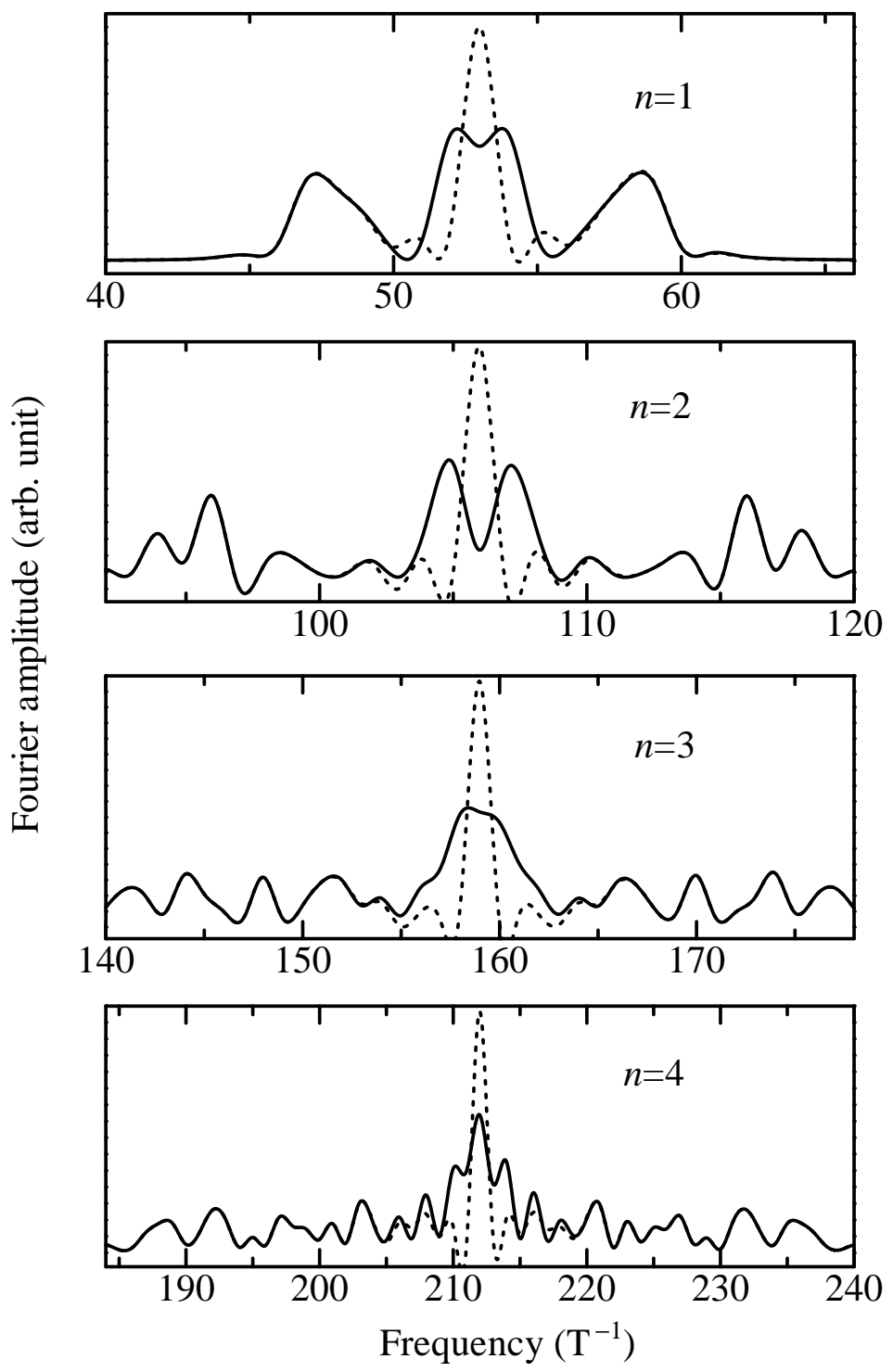

FIG. 5: Solid lines are the FT spectra of the function (5) for the winding number $n=1,2,3,4$. The magnetic field region is taken as $-0.5 \mathrm{~T}<B<0.5 \mathrm{~T}$ and the parameter $\Delta k_{\mathrm{F}}$ is taken from $\mathrm{SdH}$ measurement as $4.1 \times 10^{7} \mathrm{~m}^{-1}$. The scale of abscissa is modified with $n$ to show the entire peak structures. Dotted lines are the results when Berry's phase $\Delta \theta_{\mathrm{B}}$ is set to zero. Significant difference between solid and dotted lines around the center while they are almost identical in surrounding regions. 